\title{
Oxigenación con membrana extracorpórea neonatal-pediátrica
}

\author{
JAVIER KATTAN S., M.D. ${ }^{1}$, ÁLVARO GONZÁLEZ M., M.D. ${ }^{1}$, ANDRÉS CASTILLO M., M.D. ${ }^{2}$ \\ 1. Pediatra Neonatólogo, Sección de Neonatología, División de Pediatría, Facultad de Medicina, Pontificia Universidad \\ Católica de Chile. \\ 2. Pediatra Intensivista, Unidad Paciente Crítico Pediátrico, División de Pediatría, Facultad de Medicina, Pontificia \\ Universidad Católica de Chile.
}

\begin{abstract}
Neonatal and Pediatric exracorporeal membrane oxygenation

In 1973 and 1975 the Extracorporeal Membrane Oxygenation (ECMO) therapy was first successfully used in neonatal patients with cardiac and respiratory failure who were unresponsive to maximal therapy. ECMO allows the heart and/or lungs time to rest and heal, providing opportunity for recovery. ECMO is used in neonatal-pediatric areas as rescue therapy in over 1,200 patients with respiratory failure and about 750 patients with heart diseases every year, according to reports from the "Extracorporeal Life Support Organization" (ELSO). The best survival results in short and long term are achieved in patients with isolated respiratory diseases, becoming a standard therapy in high complexity referral centers. The first Chilean neonatal/pediatric ECMO program was started in the Catholic University Hospital in 2003, becoming the first program associated with ELSO in Latin America. In recent years, new neonatal-pediatric ECMO programs have been created in high complexity centers in several Latin American countries such as Argentina, Colombia, Brazil, Mexico and Chile, which are now giving form to the ELSO Latin American Chapter. We hope that these new ECMO referral centers, associated to a better network management, positively impact the survival of infants and children with respiratory or cardiac failure and be available in the future to a greater number of patients in our region. (Key words: Extracorporeal membrane oxygenation, ECMO, cardiopulmonary failure, pulmonary hypertension, ELSO).

Rev Chil Pediatr 2013; 84 (4): 367-378
\end{abstract}

\section{RESUMEN}

En los años 1973 y 1975 se utilizó por primera vez en forma exitosa la terapia de oxigenación con Membrana Extracorpórea (ECMO) para pacientes neonatales con falla cardíaca y respiratoria que no respondían a la terapia máxima. La ECMO da “tiempo" para el descanso pulmonar y/o cardíaco, y así da oportunidad a la recu-

Recibido el 17 de junio de 2013, aceptado para publicación el 17 de julio de 2013.

Este trabajo cumple con los requisitos sobre consentimiento /asentimiento informado, comité de ética, financiamiento, estudios animales y sobre la ausencia de conflictos de intereses según corresponda. 
peración. ECMO es utilizada en el área neonatal-pediátrica como terapia de rescate en más de 1.200 pacientes con falla respiratoria y en cerca de 750 con patologías cardíacas al año, según reportes de la "Extracorporeal Life Support Organization" (ELSO). Los mejores resultados en sobrevida a corto y largo plazo se logran en pacientes con patologías respiratorias aisladas, constituyendo hoy una terapia estándar en centros de referencia de alta complejidad. El primer Programa ECMO neonatal-pediátrico en Chile fue iniciado en el Hospital Clínico de la Pontificia Universidad Católica de Chile en el año 2003, convirtiéndose en el primer programa asociado a la ELSO en Latinoamérica. En los últimos años se han formado nuevos programas ECMO neonatales-pediátricos en centros de alta complejidad en varios países de Latinoamérica como Argentina, Colombia, Brasil, México y Chile, los cuales están fundando actualmente el Capítulo ELSO Latinoamericano. Esperamos que estos nuevos centros de referencia para ECMO, asociados a un mejor manejo en red, impacten positivamente la sobrevida de recién nacidos y niños con insuficiencia respiratoria o cardíaca y que pueda estar disponible para un mayor número de pacientes de nuestra región en un futuro cercano.

(Palabras clave: Oxigenación con membrana extracorpórea, ECMO, falla cardiopulmonar, hipertensión pulmonar, ELSO).

Rev Chil Pediatr 2013; 84 (4): 367-378

\section{Introducción}

La oxigenación con membrana extracorpórea (ECMO) o "extracorporeal life support" (ECLS) es una terapia que utiliza un "bypass" cardiopulmonar parcial modificado para dar soporte pulmonar y/o cardíaco por un tiempo prolongado, generalmente de 1 a 4 semanas (figura 1). Es utilizado en pacientes con falla cardiopulmonar reversible a causa de enfermedades pulmonares, cardíacas u otras. La ECMO da "tiempo" para el descanso pulmonar y/o cardíaco, y así da oportunidad a la recuperación. Dado que la terapia ECMO es invasiva, trae riesgos potenciales, por lo que se han diseñado criterios para seleccionar pacientes con una predicción de mortalidad entre un $50 \%$ y un $100 \%$. El candidato a ECMO ideal es el que tiene una alta predicción de mortalidad, pero con una injuria pulmonar o cardiovascular potencialmente reversible.

El primer sobreviviente adulto a la terapia ECMO fue tratado el año 1972, por J. Donald Hill, quien utilizó un oxigenador de Bramson en un paciente politraumatizado ${ }^{1}$. A finales de los años 70 esta terapia fue abandonada en adultos por sus malos resultados en estudios controlados. Sin embargo, años más tarde la ECMO resurgió en pacientes neonatales y pediátricos gracias al cirujano Robert Bartlet, quien el año 1975 en la Universidad de Michigan, trató al primer paciente neonatal sobreviviente a esta terapia, una recién nacida $(\mathrm{RN})$ latina abandonada quien padecía de un síndrome de dificultad respiratoria ${ }^{1}$. El uso en $\mathrm{RN}$ creció hacia fines de los años 80 , con sobrevidas cercanas al $80 \%$ en pacientes con una predicción de mortalidad sobre el 60-80\%. Debido a la expansión de su uso en pacientes neonatales, el año 1989 se forma una alianza voluntaria entre los centros ECMO activos y se conforma la "Extracorporeal Life Support Organization" (ELSO).

Cerca del $80 \%$ de los más de 50.000 pacientes tratados con ECMO y reportados a la ELSO son RN o niños, siendo el grupo de RN de causa respiratoria aproximadamente la mitad del total de pacientes reportados ${ }^{2}$ (figura 2).

En las últimas décadas, en parte por los progresos del cuidado intensivo neonatal-pediátrico y la mejoría del cuidado obstétricoperinatal, la mortalidad neonatal e infantil ha mejorado en muchos países de Latinoamérica, llegando por ejemplo en Chile a cifras de 5,1 y 7,4 por 1.000 nacidos vivos, respectivamente ${ }^{3}$. Sin embargo, parte importante de esta mortalidad ocurre en RN con patologías cardiorrespiratorias como el síndrome de aspiración de meconio (SAM), neumonía, sepsis, hipertensión pulmonar persistente (HPPN) grave, hernia diafragmática congénita y algunas cardiopatías congénitas. De acuerdo a datos nacionales de los Ministerios de Salud Latinoamericanos, muere cerca de $1 \mathrm{RN}$ de cada 1.000 

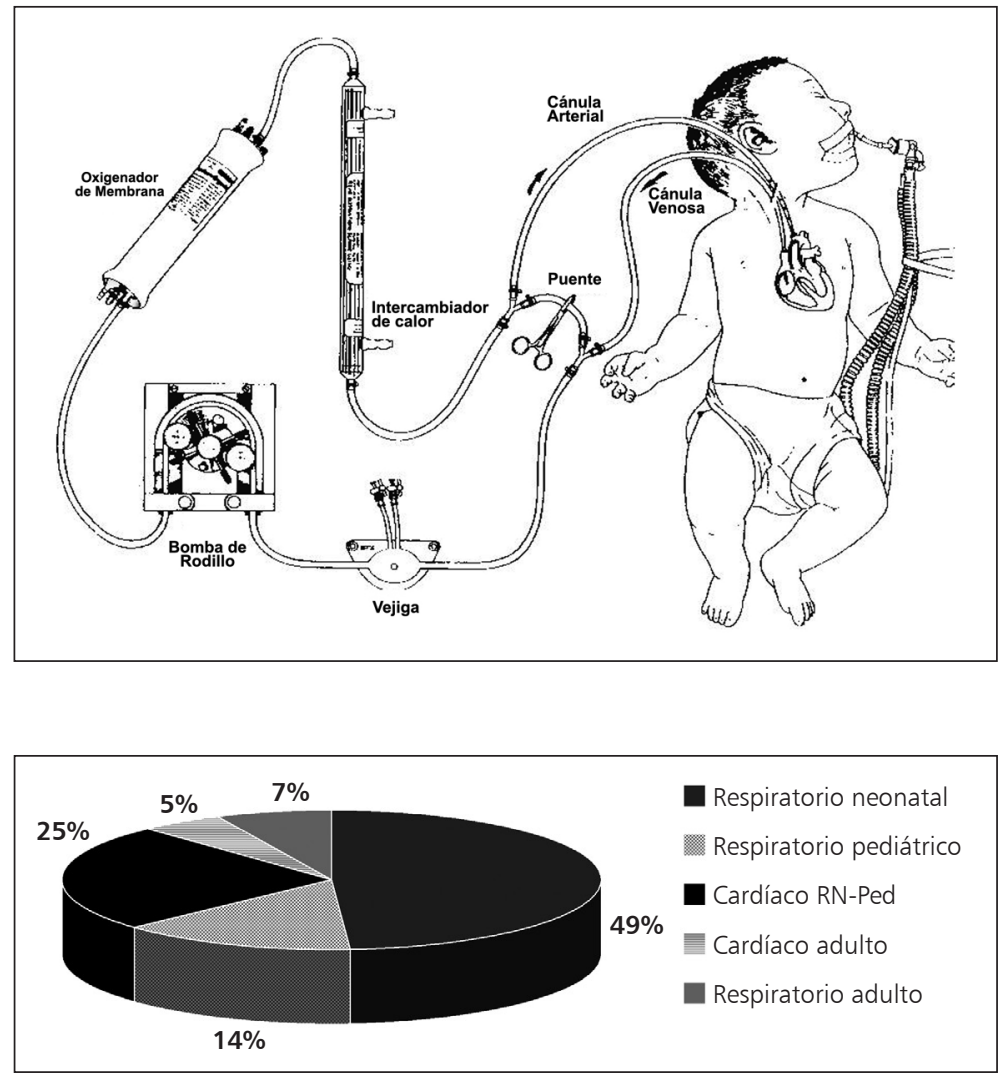

Figura 1. Esquema circuito ECMO venoarterial clásico con bomba de rodillo y oxigenador de membrana de silicona. Sangre venosa es obtenida de la aurícula derecha vía yugular interna derecha, luego bombeada, oxigenada, calentada y devuelta a la aorta vía carótida derecha. Esquema modificado, con autorización, del manual de ECMO del "Children's National Medical Center, George Washington University, Washington D.C. '.

Figura 2. Distribución de pacientes que requieren ECMO según edad y etiología de base (cardíaca o pulmonar).
RN vivos $>2.000 \mathrm{~g}$ a causa de insuficiencia respiratoria grave ${ }^{3}$.

En los años 90 aparecieron nuevas terapias para combatir las patologías cardiorrespiratorias, tales como ventilación de alta frecuencia oscilatoria (VAFO), surfactante, óxido nítrico inhalatorio (iNO) ${ }^{4}$. Con estas terapias, en asociación a centros ECMO, se ha logrado disminuir significativamente la morbimortalidad de estas patologías en los países más desarrollados.

En la última década, la oxigenación con membrana extracorpórea (ECMO) es utilizada como terapia de rescate en cerca de 800 RN reportados a la ELSO al año, quienes no respondieron al cuidado intensivo con ventilación de alta frecuencia oscilatoria (VAFO) y/o óxido nítrico inhalatorio (iNO) ${ }^{2,5}$. Actualmente en EE.UU., la tasa de uso de ECMO es de aproximadamente 1 por cada $6.000 \mathrm{RN}$ vivos. Esta terapia ha demostrado claramente, en RN con insuficiencia respiratoria grave, una mayor sobrevida global (75\% al alta), mejor calidad de vida a futuro y favorable relación costoefectividad $^{2}$ (figuras 3 y 4).

En pacientes pediátricos respiratorios, las indicaciones que llevan a ECMO son más diversas y difíciles de definir que en el período neonatal ${ }^{6}$, sin embargo, en los últimos años ha aumentado el número de casos reportados a la ELSO a cerca de 350 niños al año, con una sobrevida global al alta de un $56 \%$ (2,5,6 (figura 5). La falla respiratoria hipóxica aguda es el mecanismo fisiopatológico más frecuente de entrada a ECMO de causa respiratoria ${ }^{7}$. Dentro de este grupo, la neumonia viral es la causa más frecuente y una de las con mejor sobrevida junto con las neumonias aspirativas y el síndrome de distrés respiratorio agudo postraumático ${ }^{2}$ (figura 6). Hoy a menudo ingresan pacientes con inmunosupresión y sospecha de sepsis, quienes frecuentemente tienen falla multiorgánica ${ }^{7}$. Los grupos de pacientes pediátricos con peor pronóstico son aquellos con trasplante de 

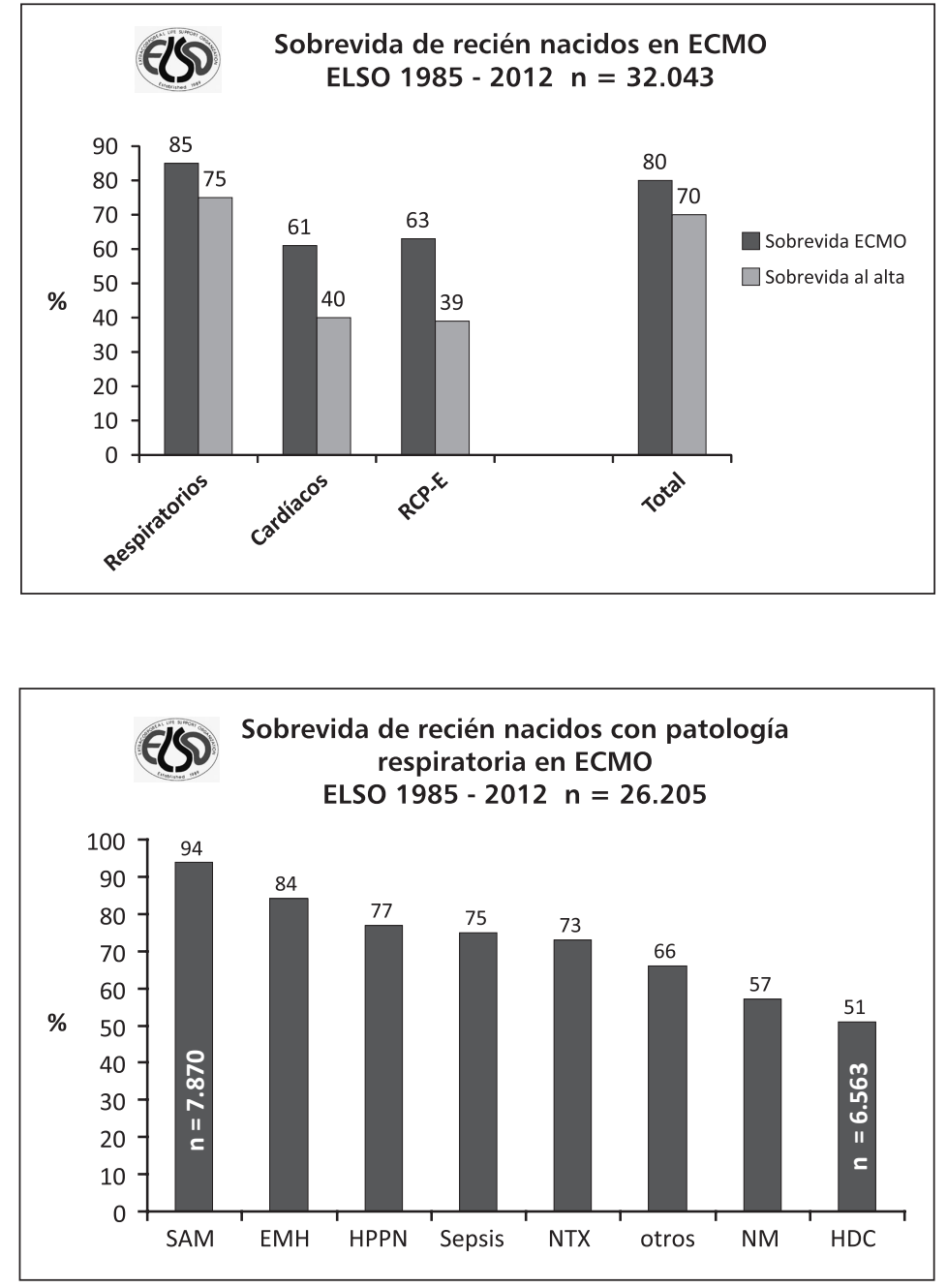

Figura 3. Sobrevida de 32.043 recién nacidos post ECMO reportados a la "Extracorporeal Life Support Organization" (ELSO), agrupados según causa de ingreso a ECMO. (RCP-E: resucitación cardiopulmonar extracorpórea).
Figura 4. Sobrevida al alta de 26.205 recién nacidos tratados con ECMO, reportados a la ELSO según causa respiratoria. SAM: síndrome aspirativo meconial, EMH: enfermedad de membrana hialina, HPPN: hipertensión pulmonar persistente neonatal, NTX: neumotórax, NM: neumonia, HDC: hernia diafragmática congénita. médula ósea, neumonía por Bordetella pertussis e hipertensión pulmonar y quienes ingresan a ECMO con falla multiorgánica, en oposición al buen pronóstico de quienes sólo tienen compromiso pulmonar aislado ${ }^{7}$.

Así como en los años 70 se utilizó ECMO para el manejo de la falla respiratoria e hipertensión pulmonar, también se inició poco más tarde la asistencia cardíaca ventricular, reportándose el año 1973 por Soeter, el primer caso de soporte cardíaco postoperatorio prolongado en un paciente con tetralogía de Fallot ${ }^{1}$. Hoy más de la mitad de los pacientes que requieren ECMO cardíaco son pacientes con cardiopatías congénitas cianóticas complejas ${ }^{2}$ (figura 7). Lideran el grupo que requiere ECMO los pacientes post cardiotomía por un canal AV completo (20\%), anatomía de ventrículo único complejo $(17 \%)$ y tetralogía de Fallot $(14 \%)^{8}$. Dentro de las causas principales que llevan a la necesidad de ECMO peri-operatorio cardíaco están la hipoxia (36\%), el paro cardíaco (24\%) y la falla a la salida de circulación extracorpórea (14\%). Por consiguiente, el uso de iNO y VAFO pueden disminuir la necesidad de ECMO al disminuir el grado de hipoxia ${ }^{8}$.

ECMO es superior a los dispositivos de asistencia ventricular en aquellos casos donde la hipoxia, la hipertensión pulmonar o la falla biventricular son los mecanismos fisiopatológicos predominantes ${ }^{8}$.

En los últimos años, la indicación cardíaca 

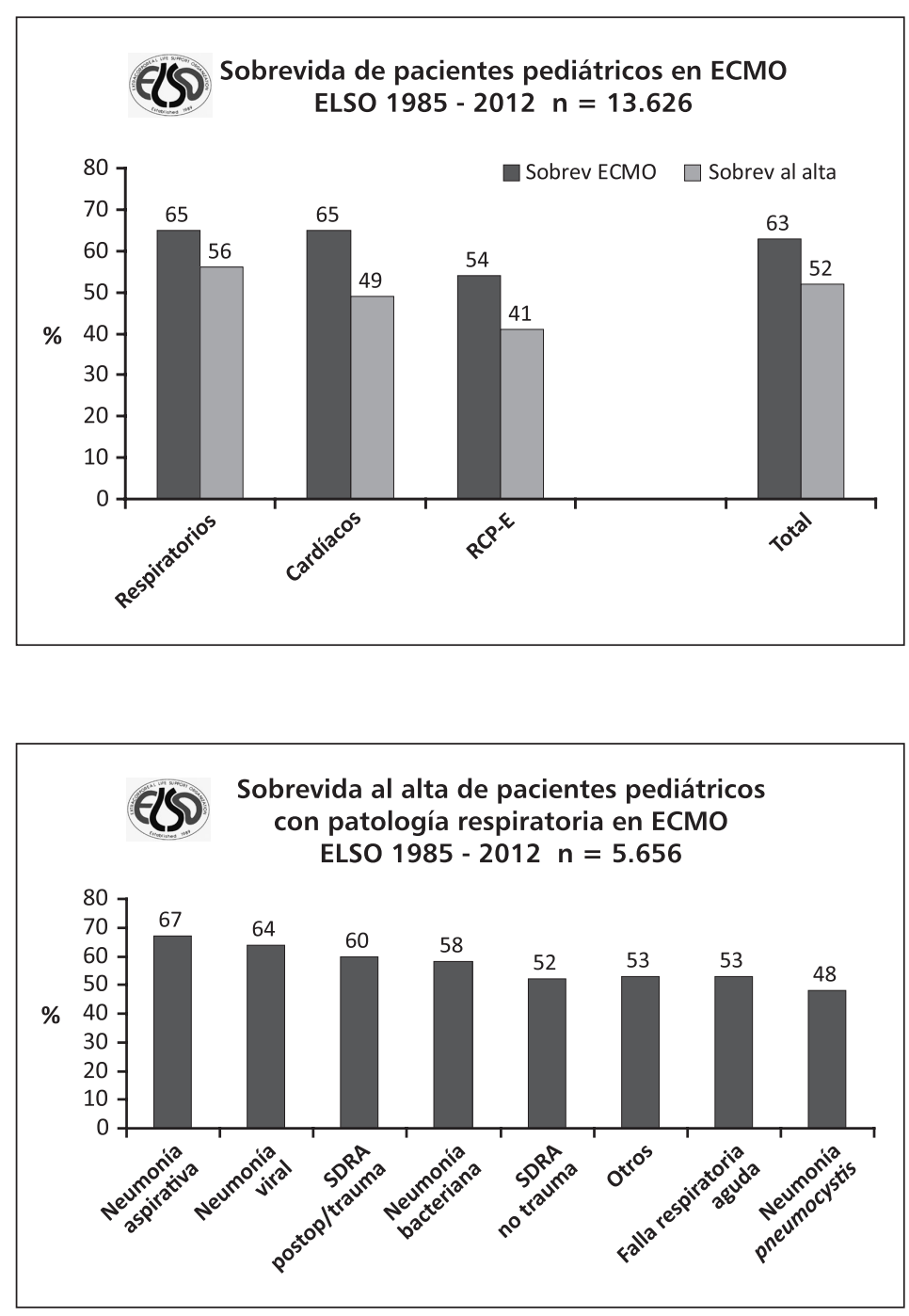

Figura 5. Sobrevida de 13.626 pacientes pediátricos post ECMO reportados a la "Extracorporeal Life Support Organization" (ELSO), agrupados según causa de ingreso a ECMO (RCP-E: resucitación cardiopulmonar extracorpórea).
Figura 6. Sobrevida al alta de 5.656 pacientes pediátricos tratados con ECMO, reportados a la ELSO según causa respiratoria. SDRA: síndrome de distrés respiratorio agudo.

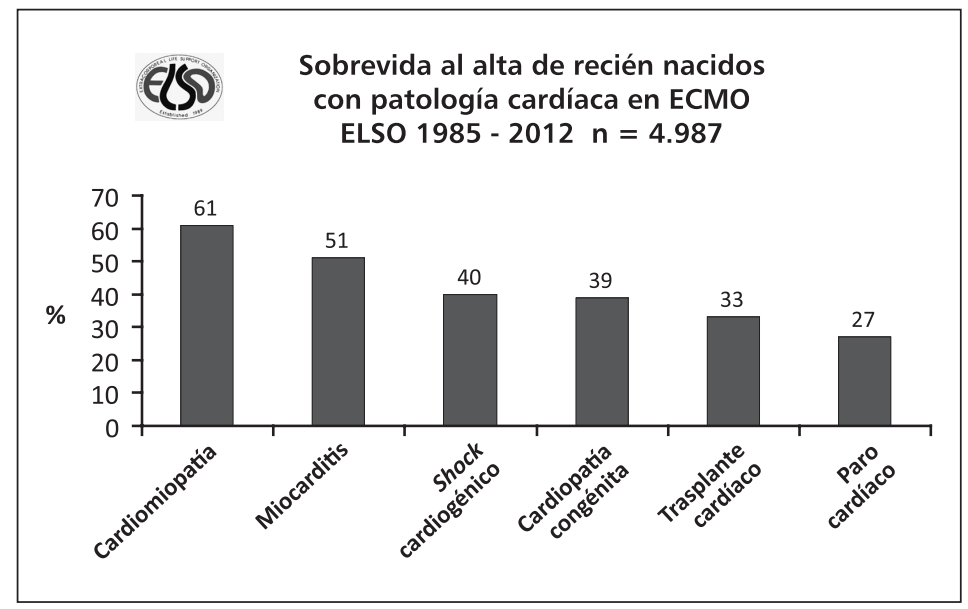

Figura 7. Sobrevida al alta de 4.987 recién nacidos tratados con ECMO, reportados a la ELSO según causa cardíaca. 
neonatal-pediátrica ha aumentado sostenidamente a más de 750 casos al año reportados a la ELSO, constituyendo una valiosa terapia de apoyo en los centros de cardiocirugía de alta complejidad $^{2}$.

\section{Fisiología ECMO}

Durante el soporte extracorpóreo la sangre es drenada desde el paciente a una bomba externa (de rodillo o centrífuga), la cual impulsa la sangre a través de un membrana de intercambio (oxigenador de silicona o polimetilpentene) para oxigenación y remoción de $\mathrm{CO}_{2}$ y a un calentador, para luego devolver la sangre a la circulación del paciente (figura 1). Esta terapia requiere de anticoagulación del circuito y del paciente mediante heparina administrada al circuito ECMO, con el fin de evitar la activación de la cascada de coagulación en el sistema. Además, se utilizan varios monitores de presión, flujo, burbujas y temperatura. Es esencial el monitoreo continuo de la coagulación mediante la medición del tiempo activado de la coagulación (ACT) en forma horaria y medición de recuento de plaquetas, TP y fibrinógeno.

Existen básicamente dos formas de ECMO:

\section{a) Venoarterial (VA)}

En el cual la sangre es drenada de la aurícula derecha, a través de una cánula inserta en la vena yugular interna derecha, vena femoral o directamente en la aurícula derecha y es retornada a la aorta torácica, a través de una cánula carotídea derecha, femoral o aórtica (figura 1). ECMO VA entrega soporte cardíaco y pulmonar. En pacientes postoperados cardíacos se utilizan frecuentemente cánulas por vía transtorácica (cánula auricular derecha y aórtica).

\section{b) Venovenoso ( $\mathrm{VV})$}

En el cual la sangre es drenada de la aurícula derecha, a través de los orificios posteriores e inferior de una cánula doble lumen insertada en la yugular derecha, y devuelta a la misma aurícula derecha, a través de los orificios anteriores de esta cánula, los que están dirigidos hacia la válvula tricúspide. Una de las limitantes de este método es la recirculación de sangre ya oxigenada a través de la cánula doble lumen, lo que se ha corregido con nuevos diseños de cánulas VV. También puede realizarse ECMO $\mathrm{VV}$, en niños mayores a través del uso de dos cánulas, tomando sangre desde la vena yugular y devolviéndola a la vena femoral. La ECMO $\mathrm{VV}$ requiere de una buena función cardíaca. Esta modalidad ECMO evita la canulación de la arteria carótida o femoral, disminuyendo así complicaciones derivadas de canular o ligar estas arterias y de la entrada de aire al circuito ECMO. Este método ha aumentado en los últimos años y se utiliza hoy en alrededor del 40 y $50 \%$ de los casos respiratorios neonatales y pediátricos, respectivamente.

Con ambas formas de ECMO, los parámetros del ventilador y la $\mathrm{FiO}_{2}$ son disminuidos para permitir la recuperación del pulmón, pero generalmente el PEEP se mantiene más alto (Ej., 6 a $8 \mathrm{~cm} \mathrm{H}_{2} \mathrm{O}$ ) para evitar atelectasias.

Durante la ECMO, la entrega de oxígeno resulta de la combinación de; la oxigenación de la sangre a través de la membrana, el flujo de sangre a través del circuito extracorpóreo, la oxigenación a través del pulmón nativo y del gasto cardíaco del corazón nativo ${ }^{1}$. A su vez, la oxigenación en la membrana ECMO es función de; la geometría de ésta, del material que se compone y su grosor, del espesor de la lámina de sangre, de la $\mathrm{FiO}_{2}$, el tiempo de permanencia de los glóbulos rojos en el área de intercambio, de la concentración de hemoglobina y la saturación de $\mathrm{O}_{2}{ }^{1}$.

Por otro lado, la remoción de $\mathrm{CO}_{2}$ en ECMO es en función de la geometría de la membrana, su material, área de superficie, $\mathrm{PCO}_{2}$ sanguínea y en menor grado depende del flujo sanguíneo y del flujo de gas a través de la membrana ${ }^{1}$.

En el ECMO VA, el bypass genera un flujo esencialmente no pulsátil. Así, en la medida que se aumenta el flujo de sangre al circuito extracorpóreo, la onda de pulso comienza a disminuir y luego al alcanzar un $100 \%$ de bypass se aplana completamente, sólo con ondas ocasionales. Sin embargo, lo habitual en ECMO VA es que sólo se alcance un bypass cercano a un $80 \%$, dejando circular un $20 \%$ o más de la sangre por el corazón izquierdo y los pulmones, resultando así en una onda de pulso disminuida, pero visible ${ }^{1}$. El riñón es sin 
duda el órgano más afectado por la falta de pulsatilidad, produciendo un efecto anti-diurético debido a la estimulación juxtaglomerular. Además, el flujo no pulsátil se ha relacionado con estimulación de los receptores de presión del seno carotídeo, provocando una gran liberación de catecolaminas con efectos deletéreos en la microcirculación ${ }^{1}$.

\section{Criterios de selección para ingreso de pacientes a ECMO}

Los criterios difieren si son pacientes neonatales o pediátricos y dependen de si la causa es primariamente cardíaca o respiratoria. Los criterios son generales y deben ser individualizados para cada paciente, evaluando los riesgos y beneficios de entrar a $\mathrm{ECMO}^{7}$ (tabla 1).

Para pacientes pediátricos con falla respiratoria, los criterios fundamentales son similares a los neonatales, colocando especial énfasis en si se está frente a una enfermedad pulmonar grave con riesgo elevado de muerte y frente a un proceso reversible mediante reposo respiratorio, gasométrico y hemodinámico (tabla 2).

Una contraindicación específica para ECMO en pacientes cardíacos es la presencia de lesiones residuales pos cirugía o la presencia de contraindicaciones para trasplante cardíaco, pero cada caso debe ser analizado individualmente ya que muchas veces existen contraindicaciones relativas o que cambian en el tiempo ${ }^{8}$. Entre las indicaciones por causa cardíaca o hemodinámica primaria en pacientes pediátricos destaca:

- Descompensación cardiovascular grave pero potencialmente reversible que no responda a drogas vasoactivas, vasodilatadores, antiarrítmicos o marcapaso.

- $\mathrm{SVO}_{2}<60 \%$; $\mathrm{pH}<7,15$ persistente.

- Falla en salir de bypass cardiopulmonar luego de cirugía.

- Arritmias graves con pobre perfusión.

- Rápido deterioro o disfunción ventricular grave.

\section{Manejo en ECMO}

Los parámetros iniciales apuntan a lograr un bypass igual o mayor a un 50\% del gasto cardíaco (gasto cardíaco estimado de $200 \mathrm{ml} /$ $\mathrm{kg} / \mathrm{min}$ ), y son ajustados para mantener una adecuada oxigenación, presión arterial y estado ácido-base. En pacientes con falla cardíaca, la ECMO VA es el método de elección. Cuando la función cardíaca está conservada y la patología principal es pulmonar, se puede utilizar ECMO VV para ayudar a la oxigenación y ventilación. La atención meticulosa de todos los aspectos del paciente es esencial. Requieren controles frecuentes de gases en sangre tanto del paciente como del circuito ECMO, controles de coagulación, función renal y evaluación ecográfica cerebral en busca de he-

Tabla 1. Criterios de selección para ingreso de neonatos a ECMO

- Edad gestacional $\geq 34$ semanas

- Peso de Nacimiento $\geq 2 \mathrm{~kg}$

- Falla al manejo médico máximo (VAFO, iNO, surfactante)

- Condición cardiopulmonar reversible

- Ventilación mecánica $\leq 10$ - 14 días

- Alta mortalidad pulmonar (50-100\%). Una de las siguientes:

- Índice de oxigenación $(\mathrm{IO})>40$ por 4 h (iNO, VAFO)

- $\mathrm{PaO}_{2}<40-50 \mathrm{mmHg}$ por $4 \mathrm{~h}\left(100 \% \mathrm{O}_{2}\right)$

- Gradiente A/aDO2 > $600 \mathrm{mmHg}$ por $4 \mathrm{~h}$

- IO $\geq 25$ luego de 72 h con VAFO-iNO ${ }^{9}$

- Acidosis metabólica inmanejable ( $\mathrm{pH}<7,15$ por 2 h)

- Gasto cardíaco disminuido con etiología reversible

- Imposibilidad de salir de bypass cardiopulmonar

- Como puente para trasplante cardíaco ${ }^{10}$

- Sin lesiones residuales post cirugía cardíaca

- Ausencia de hemorragia intracraneana mayor

- Ausencia de hemorragia incontrolable

- Sin evidencia de daño cerebral masivo

- Sin malformaciones o síndromes con pronóstico letal

Tabla 2. Criterios destacados de selección para ingreso de pacientes pediátricos a ECMO

- $10>40$ por 6 h en VMI y/o VAFO

- $10>35$ por $>12 \mathrm{~h}$

- Parámetros "dañinos" en ventilación mecánica

- Ventilación mecánica $\leq 10$ - 14 días

- Hipercapnia con $\mathrm{pH}<7.1$ por $4 \mathrm{~h}$

- Deterioro agudo con terapia óptima 
morragia intracraneana o infarto cerebral. Los pacientes se mantienen sedados, pero generalmente no paralizados. Permitiendo que ellos se muevan facilita la evaluación neurológica. En la medida que el paciente mejora, el soporte ECMO es reducido gradualmente. El paciente es decanulado cuando es capaz de tolerar un soporte ECMO mínimo ( $10 \%$ de bypass en ECMO VA) con parámetros de ventilación mecánica bajos o moderados. La duración del tratamiento ECMO es generalmente entre $5 \mathrm{y}$ 10 días en enfermedades respiratorias neonatales, siendo más prolongado en casos de hernia diafragmática congénita, neumonía bacteriana y miocarditis (10 a 11 días en promedio $)^{2}$.

\section{Complicaciones}

El procedimiento ECMO tiene riesgo variable de complicaciones como consecuencia de la gravedad de los pacientes al entrar a ECMO, del uso de anticoagulación y de los cambios en el flujo sanguíneo (flujo sanguíneo de menor pulsatilidad). Dentro de las complicaciones frecuentes está la hemorragia (sitio quirúrgico $6 \%$, pulmonar $4 \%$, gastrointestinal $2 \%)$, el infarto o hemorragia cerebral $(9 \%$ y $5 \%$ respectivamente), las convulsiones $(11 \%)$, la disfunción cardíaca (miocardio congelado $6 \%$, arritmias $4 \%$ ), la falla renal (4\%), la sepsis $(6 \%)$, la hiperbilirrubinemia (9\%), la hipertensión arterial (12\%) y la hemólisis $(13 \%)^{2}$. En el
ECMO de causa cardíaca la necesidad de drogas vasoactivas durante el soporte extracorpóreo es lejos la complicación más frecuente, seguido del sangramiento del sitio quirúrgico ${ }^{11}$.

La hemorragia intracraneana es la primera causa de muerte durante la ECMO, y la aparición de convulsiones es un signo de mal pronóstico. Además, existen complicaciones derivadas de la falla del circuito, del oxigenador o de los equipos ECMO.

\section{Pronóstico y programas ECMO en Latinoamérica}

La sobrevida post ECMO en pacientes neonatales varía según la enfermedad de base, siendo las causas respiratorias las con mejor resultado, cercano al 75\% de sobrevida al alta ${ }^{2}$ (figuras 3, 4 y 8). Dentro de éstas, la aspiración de meconio es la patología con mejor sobrevida en el grupo neonatal y a todas las edades, con una sobrevida al alta de un $94 \% \%^{2,12}$ (figuras 4 y 8). El tipo de ECMO para el SAM es generalmente venovenoso, lo que se asocia a una menor tasa de riesgos y complicaciones como infartos cerebrales y convulsiones, y a menores cambios en el patrón de flujo sanguíneo. Por otro lado, el grupo de pacientes que ingresa a ECMO por causa cardíaca tiene una sobrevida menor, cercana al $40 \%$, sin embargo, en pacientes bien seleccionados es

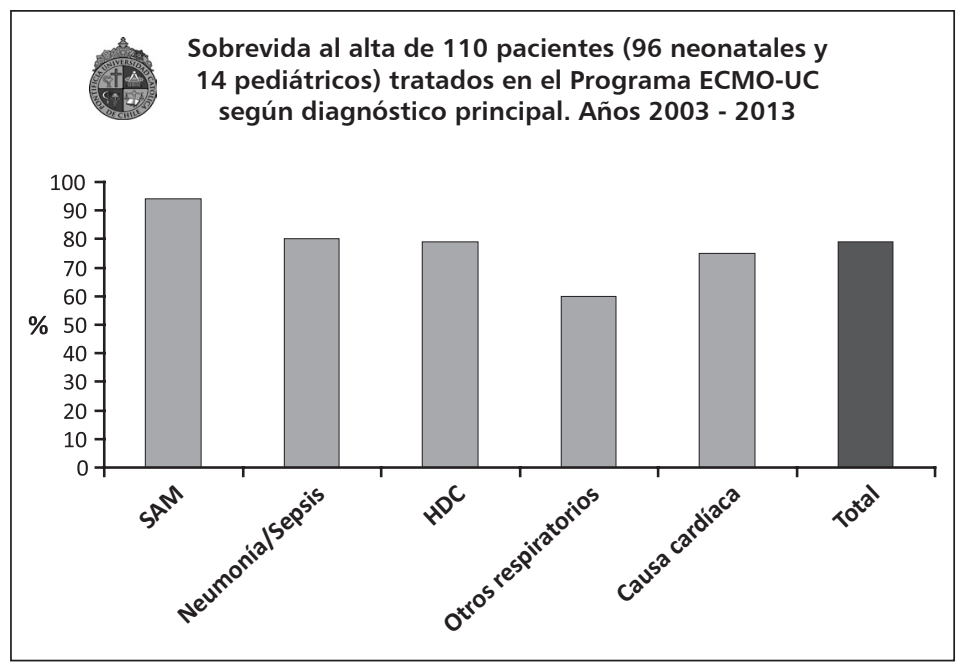

Figura 8. Sobrevida al alta de 96 pacientes neonatales y 14 pediátricos tratados en el Programa ECMO Neonatal-Pediátrico del Hospital Clínico de la Pontificia Universidad Católica de Chile (ECMO-UC) 2003-2013, reportados a la ELSO según diagnóstico principal. SAM: síndrome aspirativo meconial, HDC: hernia diafragmática congénita. 
una herramienta útil que debe estar disponible en centros cardiológicos de alta complejidad ${ }^{2}$ (figuras 3, 5 y 7). Dentro del grupo que entra a ECMO por causa cardíaca destaca la sobrevida al alta de los pacientes con cardiomiopatía y miocarditis, 61 y 51\% respectivamente ${ }^{2}$. En los últimos años se ha utilizado la ECMO como herramienta de reanimación cardiopulmonar post paro con resultados variables, cercanos a un $40 \%$ de sobrevida ${ }^{2}$ (figuras 3 y 5).

Los RN son el único grupo etario en que la terapia ECMO ha demostrado ser muy superior a la terapia convencional máxima mediante un estudio multicéntrico, controlado y randomizado, donde participaron $185 \mathrm{RN}$ con insuficiencia respiratoria grave de 55 hospitales del Reino Unido ${ }^{13,14}$. Este estudio demuestra que la mortalidad o discapacidad severa evaluada al año 1, 4 y 7 de vida disminuye significativamente al utilizar ECMO (59\% grupo terapia convencional versus $37 \%$ grupo ECMO) ${ }^{13-16}$. A los 7 años de seguimiento el $76 \%$ de los niños tenían un desarrollo cognitivo normal ${ }^{15}$.

Las revisiones sistemáticas más recientes demuestran que el uso de ECMO en RN cercanos a término con falla respiratoria severa, pero potencialmente reversible, mejora significativamente la sobrevida sin aumentar la discapacidad severa y es costo efectivo al ser comparado con otras terapias de cuidados intensivos ${ }^{17,18}$. Respecto al uso de ECMO como rescate en hernia diafragmática congénita (HDC) con falla respiratoria grave, la evidencia de los estudios prospectivos controlados muestra una reducción sólo en la mortalidad precoz $^{13,19}$. Sin embargo, un metaanálisis de los estudios retrospectivos y nuestra experiencia reportada muestran una mayor sobrevida a corto y largo plazo para HDC en las unidades que disponen de $\mathrm{ECMO}^{19,20}$.

La sobrevida y pronóstico neurológico a los 5 años en pacientes que estuvieron en ECMO de causa no cardíaca es en general muy bueno, pero empeora a menor edad gestacional, a menor peso de nacimiento y a mayor índice de oxigenación (IO) pre-ECMO ${ }^{21}$. El peor resultado en sobrevida y evolución neurológica lo tienen los pacientes con diagnóstico de shock séptico y HDC, sin embargo, son los factores pre-existentes y la gravedad del RN al entrar a ECMO los que parecen ser los grandes determinantes del pronóstico neurológico a largo plazo ${ }^{21,22}$.

El pronóstico respiratorio a largo plazo depende de la etiología de base, del grado de barotrauma y duración de la exposición a oxígeno. Entre un 10 y $30 \%$ de los pacientes con HDC presentan a los 10 años de edad episodios de sibilancias y cerca de un 50\% tienen hiperinsuflación y episodios de obstrucción de la vía aerea ${ }^{23}$.

La sobrevida post ECMO pediátrico es menor comparada con el ECMO neonatal, sin embargo destaca un mejor pronóstico en el grupo con falla respiratoria, especialmente en los pacientes con neumonía aspirativa, neumonía viral y síndrome de distrés respiratorio agudo posoperatorio o traumático ${ }^{24,25}$ (figura 6). La neumonía viral es la causa más común de ECMO pediátrico y dentro de sus etiologías la neumonía por virus respiratorio sincicial posee la sobrevida más alta post ECMO con una cifra de un $70 \%{ }^{24}$. Por otro lado, la neumonía por otros virus y por Bordetella pertussis tiene una sobrevida menor reportada de un 56\% y $39 \%$, respectivamente ${ }^{24,25}$.

El grupo pediátrico que entra a ECMO por causa cardíaca posee una sobrevida algo superior al grupo neonatal de causa cardíaca ${ }^{2}$ (49\% sobrevida al alta), destacando la sobrevida de la miocarditis y cardiomiopatía con cifras al alta de un 73 y un $57 \%$, respectivamente ${ }^{2}$.

Dada la evidencia que demuestra un claro beneficio en sobrevida, calidad de vida y costoefectividad de esta terapia y ante la ausencia de un Programa formal de ECMO en Chile, se decidió en el año 1998 constituir un Programa ECMO Neonatal-Pediátrico en la Unidad de Neonatología del Hospital Clínico de la Pontificia Universidad Católica de Chile (ECMO-UC) según las normas establecidas por la ELSO (Extracorporeal Life Support Organization), para pacientes que presenten insuficiencia respiratoria o cardiovascular grave reversible, refractaria al tratamiento convencional máximo ${ }^{26}$.

Desde el año 1999 se inicia la preparación de un equipo multidisciplinario (neonatólogos, intensivistas pediátricos, cirujanos cardíacos e infantiles, enfermeras, perfusionistas, terapistas respiratorios, psicólogas) con entrenamiento en centros ECMO de EE.UU. afiliados 
a la $\mathrm{ELSO}^{26}$. Paralelamente se adquirieron los equipos para conformar las primeras unidades de ECMO según recomendaciones de la ELSO. El entrenamiento se consolidó en Chile con un curso experimental en corderos. Se establecieron como criterios fundamentales de selección la reversibilidad de la patología respiratoria o cardíaca, la falla a la terapia máxima convencional, un peso $\geq 2 \mathrm{~kg}$, edad gestacional $\geq 34$ semanas, índice de oxigenación $>40$, ventilación mecánica $<14$ días y ausencia de lesión cerebral grave o falla multiorgánica ${ }^{26}$.

Luego de 10 años, se ha consolidado el primer Programa de ECMO Neonatal-Pediátrico en Chile, primer centro Latinoamericano miembro de la ELSO (Figura 9). Desde el año 2003 al año 2013 se han ingresado 110 pacientes (96 RN y 14 niños) a nuestro programa ECMO, tanto por patología respiratoria como cardíaca grave (rango edad de 0 días a 11 años (figura 8). Un 79\% (87/110) de ellos sobrevivieron al alta y están actualmente en seguimiento. Los 23 pacientes que fallecieron tenían como enfermedad de base: hernia diafragmática congénita $(\mathrm{n}=10)$, cardiopatía congénita operada con falla a la salida de circulación extracorpórea o arritmias $(\mathrm{n}=7)$, hipertensión pulmonar persistente secundaria a sepsis, síndrome aspirativo meconial o sin causa definida $(\mathrm{n}=5)$ y neumonia por Bordetella pertussis $(\mathrm{n}=1)$. Dentro del grupo tratado con ECMO, destaca un grupo importante de niños con hernia diafragmática congénita con una sobrevida al alta de un 79\% (37/47).

Al comparar el período antes y después del establecimiento de este programa ECMO en Chile, se encontró que la sobrevida del grupo total de $\mathrm{RN}$ con insuficiencia respiratoria grave $(\mathrm{IO}>25)$ aumentó significativamente desde un $75 \%(75 / 100)$ en el período pre-ECMO a un 91\% (67/74) en el período ECMO, especialmente en el grupo de $\mathrm{RN}$ con hernia diafragmática congénita ${ }^{27}$ (HDC). Durante el período ECMO, un $70 \%$ de estos pacientes con insuficiencia respiratoria grave fueron rescatados con iNO y/o VAFO, mientras que un 30\% no mejoraron, ingresando a ECMO un $76 \%$ de estos últimos ${ }^{27}$.

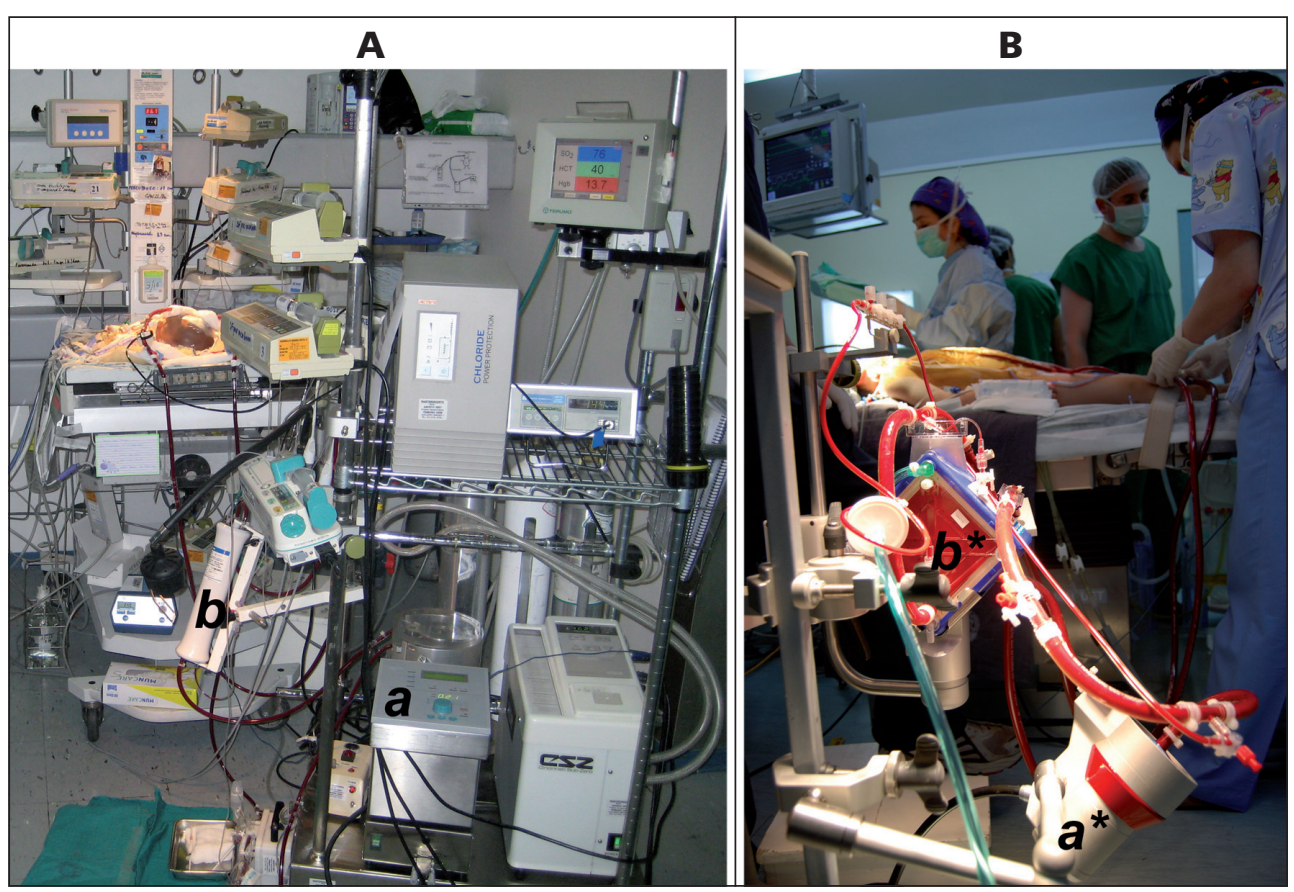

Figura 9. Dos pacientes del Programa ECMO Neonatal-Pediátrico del Hospital Clínico de la Pontificia Universidad Católica de Chile. A. Paciente neonatal respiratorio en ECMO mediante bomba de rodillo (a) y oxigenador de membrana de silicona (b). B. Paciente pediátrico cardíaco en ECMO mediante bomba centrífuga (a) y oxigenador de polimetilpentene (b). 
El $100 \%$ de los sobrevivientes de nuestro programa está actualmente en un programa especial de seguimiento post ECMO. Dentro del seguimiento neurológico, el Test de Bayley II a los 12-18 meses, muestra que sobre el 90\% de los niños tiene índice de desarrollo mental (MDI) normal o levemente alterado y un índice de desarrollo psicomotor (PDI) normal o con alteración leve en más del $70 \%{ }^{28}$. Además, ningún paciente presenta alteraciones visuales $o$ auditivas invalidantes.

Respecto al seguimiento respiratorio, el $83 \%$ de los pacientes tiene evaluación clínica broncopulmonar normal o levemente alterada a los 12-18 meses $^{28}$.

El establecimiento de un programa ECMO en Chile se asoció a un aumento significativo en la sobrevida de RN cercanos a término con insuficiencia respiratoria grave. La terapia ECMO fue exitosa y no provocó secuelas invalidantes en la mayoría de los pacientes.

Así como también en Chile, en los últimos años se han formado nuevos programas ECMO neonatales-pediátricos en centros de alta complejidad y alto volumen de pacientes en varios países de Latinoamérica como Argentina, Colombia, Brasil y México, la mayoría de los cuales han ingresado progresivamente a la ELSO y están fundando actualmente el Capítulo ELSO Latinoamericano.

Esperamos que estos nuevos programas ECMO como centros de referencia, asociados a un mejor manejo médico en red, impacten positivamente la sobrevida de RN y niños con insuficiencia respiratoria o cardíaca y que pueda estar disponible para un mayor número de pacientes de nuestra región en un futuro cercano.

\section{Conclusiones}

La terapia ECMO, hoy llamada en forma más amplia "extracorporeal life support" (ECLS) es una terapia estándar en neonatología y pediatría, con demostrados beneficios a corto y largo plazo. Es posible incorporarla a la práctica de terapia intensiva con buenos resultados en países en vías de desarrollo, pero requiere ser implementada en centros neona- tales y pediátricos de alta complejidad, que cuenten con personal entrenado y con un alto nivel de compromiso.

En el futuro, los pacientes que se beneficien con ECLS serán progresivamente más complejos, por lo que se requerirá de nuevas modalidades de ECLS más simples, automáticas y con menor necesidad de anticoagulación, con el fin de minimizar sus riesgos y hacer posible su uso más prolongado. Así podrán ingresar RN y niños muy graves a ECLS en espera de un trasplante cardíaco o pulmonar ${ }^{10}$, o como puente a dispositivos de asistencia ventricular. Incluso RN prematuros con falla cardiopulmonar grave se podrían beneficiar en el futuro con ECLS parciales por vía umbilical. Nuevos oxigenadores microporosos de baja resistencia podrían prescindir del uso de una bomba, donde la arteria y vena umbilical se usarían como shunt arteriovenoso. Además, RN con HDC podrían ingresar a ECLS en forma precoz para minimizar su daño pulmonar y favorecer el crecimiento pulmonar utilizando, por ejemplo, factores de crecimiento y/o ventilación líquida con perfluorocarbono asociados a ECLS.

De esta manera, esperamos que ECLS nos permita seguir asistiendo la función pulmonar y/o cardíaca en forma más racional, mientras se produce la reparación cardiopulmonar de procesos graves, pero reversibles.

\section{Referencias}

1.- Bartlett R: Physiology of ECLS. In: ECMO: Extracorporeal cardiopulmonar support in critical care, $4^{\text {th }}$ edition Michigan. Editors: Annich GM, Lynch WR, MacLaren G, Wilson JM, Bartlett RH: 11-31, 2012.

2.- ELSO: ECMO Registry of the Extracorporeal Life Support Organization (ELSO), Ann Arbor, Michigan, 2013.

3.- Gobierno de Chile, Ministerio de Salud, Departamento de Estadística e Información de Salud: Defunciones de menores de un año, por algunos grupos de causas específicas de muerte y por servicio de salud 2004 y 2010. Estadística de Natalidad y Mortalidad 2004 y 2010.

4.- Kinsella JP, Truog WE, Walsh WF, et al: Randomized, multicenter trial of inhaled nitric oxide and highfrequency oscillatory ventilation in severe, persistent pulmonary hypertension of the newborn. J Pediatr 1997; 131: 55-62. 
5.- Conrad SA, Rycus, PT, Dalton H: Extracorporeal life support registry report 2004. Asaio J 2005; 51: 4-10.

6.- Segura S, Cambra FJ, Moreno J, et al: Experiencia en edad pediátrica. An Pediatr (Barc) 2009; 70 (1): 12-9.

7.- Bohn D: Acute hypoxic respiratory failure in children. In: ECMO: extracorporeal cardiopulmonary support in critical care, $4^{\text {th }}$ edition Michigan. Editors: Annich GM, Lynch WR, MacLaren G, Wilson JM, Bartlett RH: 4172, 2012.

8.- Thiagarajan RR, Yarlagadda V, Bratton SL: Cardiac failure: principles and physiology. In ECMO: extracorporeal cardiopulmonar support in critical care, $4^{\text {th }}$ edition Michigan. Editors: Annich GM, Lynch WR, MacLaren G, Wilson JM, Bartlett RH: 33-39, 2012.

9.- Kössel H, Bauer K, Kewitz G, et al: Do we need new indications for ECMO in neonates pretreated with high-frequency ventilation and/or inhaled nitric oxide? Intensive Care Medicine 2000; 26: 1489-95.

10.- Bae JO, Frischer JS, Waich M, Addonizio LJ, Lazar EL, Stolar CJ: Extracorporeal membrane oxygenation in pediatric cardiac transplantation. J Pediatr Surg 2005; 40 (6): 1051-6.

11.- Haines NM, Rycus PT, Zwischenberger JB, Bartlett RH, Undar A: Extracorporeal Life Support Registry Report 2008: neonatal and pediatric cardiac cases. ASAIO J 2009; 55 (1): 111-6.

12.- Short BL: Extracorporeal membrane oxygenation: use in meconium aspiration syndrome. J Perinatol 2008; 28 Suppl 3: S79-83.

13.- UK, CETG: UK collaborative randomized trial of neonatal extracorporeal membrane oxygenation. Lancet 1996; 348: 75-82.

14.- Bennett CC, Johnson A, Field DJ, Elbourne D: UK collaborative randomized trial of neonatal extracorporeal membrane oxygenation: follow-up to age 4 years. Lancet 2001; 357: 1094-6.

15.- McNally H, Bennett CC, Elbourne D, Field DJ: United Kingdom collaborative randomized trial of neonatal extracorporeal membrane oxygenation: follow-up to age 7 years. Pediatrics 2006; 117: e845-54.

16.- Petrou S, Edwards L: Cost effectiveness analysis of neonatal extracorporeal membrane oxygenation based on four year results from the UK Collaborative ECMO Trial. Arch Dis Child Fetal Neonatal Ed 2004; 89: F263-8.

17.- Mugford M, Elbourne D, Field D: Extracorporeal membrane oxygenation for severe respiratory failure in newborn infants. Cochrane Database Syst Rev 2008; 16
(3): CD001340.

18.- Pawlik TD, Porta NF, Steinhorn RH, Ogata E, deRegnier $R A$ : Medical and financial impact of a neonatal extracorporeal membrane oxygenation referral center in the nitric oxide era. Pediatrics 2009; 123 (1): e17-24.

19.- Morini F, Goldman A, Pierro A: Extracorporeal membrane oxygenation in infants with congenital diaphragmatic hernia: a systematic review of the evidence. Eur J Pediatr Surg 2006; 16 (6): 385-91.

20.- Kattan J, Godoy L, Zavala A, et al: Improvement of survival in infants with congenital diaphragmatic hernia in recent years: effect of ECMO availability and associated factors. Pediatr Surg Int 2010; 26 (7): 671-6.

21.- Karimova A, Brown K, Ridout D, et al: Neonatal extracorporeal membrane oxygenation: practice patterns and predictors of outcome in the UK. Arch Dis Child Fetal Neonatal Ed. 2009; 94 (2): F129-32.

22.- Davis PJ, Firmin RK, Manktelow B, et al: Long-term outcome following extracorporeal membrane oxygenation for congenital diaphragmatic hernia: the UK experience. J Pediatr 2004; 144: 309-15.

23.- Hamutcu R, Nield TA, Garg M, Keens TG, Platzker AC: Long-term pulmonary sequelae in children who were treated with extracorporeal membrane oxygenation for neonatal respiratory failure. Pediatrics 2004; 114 (5): 1292-6.

24.- Zabrocki LA, Brogan TV, Statler KD, Poss WB, Rollins $M D$, Bratton SL: Extracorporeal membrane oxygenation for pediatric respiratory failure: survival and predictors of mortality. Crit Care Med 2011; 39: 364-70.

25.- Smalley N, MacLaren G, Best D, Paul E, Butt W: Outcome in children with refractory pneumonia supported with extracorporeal membrane oxygenation. Intensive Care Medicine 2012; 38: 1001-7.

26.- Kattan J, González A, Becker P, et al: Extracorporeal membrane oxygenation (ECMO): consolidation of a neonatal-pediatric program in Chile and report of three cases. Rev Med Chile 2005; 133: 1065-70.

27.- Kattan J, González A, Becker P, et al: Survival of Newborn Infants With Severe Respiratory Failure Before and After Establishing an Extracorporeal Membrane Oxygenation Program. Pediatr Crit Care Med 2013 Jul 16 [Epub ahead of print].

28.- Kattan, J, Guerrero X, Saldias MI, et al: Seguimiento de los recién nacidos graduados del primer programa neonatal de oxigenación con membrana extracorpórea en Chile. VIII Congreso Iberoamericano de Neonatología, Santiago, Chile, 2011. 